\title{
Le discours indirect libre comme patron de la prose fictionnelle de la première modernité (XVIIe-XVIII siècles)
}

\author{
Suzanne Duval \\ Université de Lausanne, Quartier Unil-Chamberonne. Section de français, 1015 Lausanne
}

\begin{abstract}
Résumé. L'étiquette de discours indirect libre existe depuis la fin du XIX ${ }^{\mathrm{e}}$ siècle, au moment où cette forme est repérée dans des textes fictionnels de la même époque. Elle n'entre pas dans les catégories rhétoriques de la représentation de la parole utilisées par les rhéteurs de l'Ancien Régime. Nous proposons une méthode pour aborder les formes des textes fictionnels des $\mathrm{XVII}^{\mathrm{e}}$ et $\mathrm{XVIII}^{\mathrm{e}}$ siècles apparentées au discours indirect libre. Nous montrons qu'il s'agit d'un patron stylistique disponible à l'époque de la première modernité dans la mesure où les traits qui le définissent forment une configuration remarquable, caractérisée par sa bivocalité, des traits stylistiques attribués à une instance autre que la voix narrative, un style bref dit " coupé » et une tendance à la désactualisation. Une telle configuration répond à une intention pragmatique de résumé et de caractérisation.
\end{abstract}

\begin{abstract}
The pattern of free indirect speech in fictional prose in the early modern age (17th-18th century)

Free indirect speech was first theorised at the end of the 19th century. It was seen (and is still often seen today) as a formal device typical of the subjectivist novel which developed at the time. There was no such category in early-modern rhetoric. Yet there was such a thing in 17th and 18th-century fiction. This paper argues that it was an available stylistic pattern in earlymodern French literature, which recognisable features were the use of a double enunciative anchorage, utterances in a voice other than that of the narrator, short sentences known as style coupé and a tendency to inactuality. This configuration served a practical purpose - that of summarising and characterising in order to show the effects of reported speech.
\end{abstract}

Le discours indirect libre (désormais DIL) est un phénomène langagier dont la théorisation est relativement récente : repéré, comme le rappellent Gilles Philippe et Joël Zufferey ${ }^{\mathrm{i}}$, à la fin du XIX ${ }^{\mathrm{e}}$ siècle, il est rapidement perçu comme une forme de prédilection du roman subjectiviste qui prend son essor à la fin du XIX ${ }^{\mathrm{e}}$ siècle ${ }^{\mathrm{ii}}$. Qu'il soit défini comme un phénomène de bivocalité disponible pour l'ensemble des pratiques discursives ${ }^{\mathrm{iii}}$, ou plutôt comme une représentation impersonnelle ou non communicationnelle du discours autre ${ }^{\mathrm{iv}}$, la forme du DIL semble appartenir, pour ce qui concerne le discours littéraire, à une culture post-rhétorique de la littérature. On pourra donc se demander à quel besoin langagier répondent les formes apparentées au DIL des textes fictionnels de la première modernité, et si elles peuvent ou non être assimilées à celles que l'on rencontre dans les corpus littéraires des $\mathrm{XIX}^{\mathrm{e}}$ et $\mathrm{XX}^{\mathrm{e}}$ siècles.

Nous préciserons dans un premier temps notre cadre méthodologique pour aborder un objet stylistique qui ne va pas de soi, le discours indirect libre étant plutôt considéré comme une forme emblématique de la littérature postérieure au XVIII ${ }^{\mathrm{e}}$ siècle ; nous rappellerons ensuite le statut du discours rapporté dans les rhétoriques et les grammaires de l'Ancien Régime afin de spécifier les cadres théoriques qui informent la pratique des auteurs de l'époque. Nous analyserons enfin quelques occurrences de discours rapportés 
présentes dans des textes narratifs des XVII ${ }^{\mathrm{e}}$ et du XVIII ${ }^{\mathrm{e}}$ siècles. Enfin, nous proposerons une interprétation pragmatique de ces occurrences.

\section{1-Discours indirect libre et stylistique du patron}

Dès l'époque de Bally, la présence de formes de DIL chez quelques auteurs des XVII et XVIII siècles fut soulignée et considérée comme une rareté, qui prouvait la valeur et la modernité d'auteurs tels que La Fayette, La Fontaine ou Rousseau ${ }^{v}$. Il manque cependant à ce jour une étude systématique de la présence ou non de cette forme dans les corpus en prose de l'Ancien Régime. Le résultat que donnerait cette enquête, quel que soit le nombre d'occurrences (abondant ou chétif) recueillies, serait précieux dans la mesure où il permettrait de savoir dans quelle mesure le DIL, souvent perçu comme une forme d'écriture typiquement littéraire et historiquement datée, est disponible pour d'autres genres et pratiques du discours. Il serait donc utile pour mieux comprendre la place du DIL dans le système des discours rapportés, la question de savoir si ce dernier n'est qu'une variante mixte des discours directs et indirects ou une forme à part entière étant encore débattue ${ }^{\text {vi }}$.

Dans un article qui se propose de mettre au jour les principes méthodologiques de la stylistique historique, Gilles Philippe insiste sur la nécessité pour une telle discipline de procéder au repérage et à l'analyse des « patrons stylistiques ${ }^{\mathrm{vii}}$ » qui informent les corpus étudiés. Selon le même auteur et Dominique Maingueneau, de tels patrons se définissent comme « la représentation imaginaire d'un type de production langagière » dont « la tradition littéraire a figé les spécificités en une sorte de stéréotype ${ }^{\text {viii }}$ ». La référence à un patron stylistique établit dans cette perspective les conditions de lisibilité d'un texte littéraire, en permettant au lecteur de saisir des faisceaux de traits de style convergents lui permettant de reconnaitre un type d'écriture.

Le terme de patron nous semble pertinent pour aborder la question des pratiques du DIL. En effet, il est possible de considérer que le DIL possède à la fois des traits formels stabilisés en langue et des traits stylistiques variables, déterminés par le contexte de sa pratique. Au XIX ${ }^{\mathrm{e}}$ siècle, les occurrences de DIL se présentent à chaque fois comme des faisceaux de traits au conventionnement instable : on rencontrera selon le cas des DIL avec ou sans déictiques ou modalisations marquées, avec ou sans nom propre... Autant de variations qui montrent que s'il s'agit bien d'une forme synthétiquement reconnaissable à la lecture, sa configuration textuelle est pourtant sujette à variation (au même titre, du reste, que celles du discours direct et indirect), et qu'elle s'inscrit dans un imaginaire de la langue qui varie en fonction des époques.

\section{2-L'imaginaire des discours rapporté sous l'Ancien Régime}

Comme l'a montré Laurence Rosier ix , la première description grammaticale du couple discours direct vs discours indirect est attestée dans la Grammaire générale et raisonnée de Port-Royal. Le passage intervient dans un chapitre consacré à l'infinitif. Les grammairiens font remarquer que la proposition infinitive latine correspond à une complétive en français :

Cette maniere de joindre les propositions par un infinitif, ou le quod \& le que, est principalement en usage quand on rapporte les discours des autres : comme si je veux rapporter que le Roy m'a dit. Je vous donneray une charge : je ne feray pas ordinairement ce rapport en ces termes, en laissant les deux Propositions separées, l'une de moy, \& l'autre du Roy : mais je les joindray ensemble par un que. Le Roy m'a dit qu'il me donnera une charge. Et alors comme ce n'est plus qu'une Proposition qui est de moy, je change la premiere personne, je donneray ; en la troisieme, il donnera, \& le pronom vous qui me signifioit le Roy parlant, au pronom me qui me signifie moy parlant ${ }^{\mathrm{x}}$.

Selon Laurence Rosier, la préférence marquée pour le discours indirect s'explique par un souci de clarté et de cohérence énonciative ${ }^{\mathrm{xi}}$. Cette réticence des grammairiens de Port-Royal envers le discours direct a aussi trait à la place de cette forme du discours rapporté dans l'imaginaire rhétorique de l'époque. En effet, le discours direct est alors considéré comme une figure de dialogisme ou sermocination. Répertoriée parmi les figures de pensée, cette figure est présentée comme une feinte énonciative : l'orateur, à la manière d'un acteur, imite le discours d'un autre que lui comme si ce dernier prenait la parole. Un tel procédé est implicitement apparenté aux genres de discours fictionnels. Sous la plume de Pierre Fabri, l'utilisation des verbes «reciter » et « contrefaire » pour caractériser le dialogisme montre bien que celui-ci est associé à une pratique langagière théâtrale et orale :

Sermocination se faict, quant l'en s'applicque a parler proprement, comme deux ou plusieurs personnes, ainsy que l'en voit souvent par dyalogue. [...]

Imitation de mœurs se faict en contrefaisant les gestes, ou en recitant les parolles et maniere de parler d'aultruy $[\ldots]$. Et ce convient assez a sermocination ${ }^{\text {xii }}$. 
Une telle figure s'éloigne de l'idéal de vérité et de naturel promu aux siècles classiques, et son usage se justifie par le caractère poétique ou récréatif du texte au sein duquel elle s'insère. La représentation du discours d'autrui est en effet considérée, en particulier dans les rhétoriques jésuites, comme un ornement rhétorique de la narration. Pour Nicolas Caussin, les « paroles » (orationes $^{\text {xiii }}$ ) des personnages participent de la vraisemblance du récit.

Les manuels de pédagogie jésuite invitent les élèves de rhétorique à amplifier leurs textes narratifs en enchâssant des discours directs. Pour le Père Pomey, les principaux ornements qui participent de la « douceur » (suavis) de la narration sont les « expressions élégantes » (verbis elebantibus), les " passions » (motus animi), les «dialogues» (Colloquentes personas) et le «dénouement inattendu » (exitus inopinati $^{\mathrm{xiv}}$ ). L'incise « dit-il » (inquit) est également considérée comme un ornement : elles appartient à la catégorie des " particules » (connectendi particulas ${ }^{\mathrm{xv}}$ ) qui permettent de lier le dialogue à la narration.

De tels préceptes s'inscrivent dans 1'«imaginaire de la liaison » qui favorise, comme l'a montré Gilles Siouffi ${ }^{\text {xvi }}(2010: 165)$, la cohérence et la cohésion de l'énoncé : sont donc privilégiées les formes du discours rapporté énonciativement unifiées et cohérentes, ce qui, a priori, ne ménage guère de place à la forme du discours indirect libre. En combinant les voix de deux énonciateurs, en effet, le DIL perd sur les deux tableaux de la valeur ornementale et dramatique du discours direct, et de la cohérence énonciative du discours indirect. La bivocalité du DIL semble en outre mal s'accorder au paradigme oral de l'énonciation qui prévaut dans les textes littéraires de l'Ancien Régime. À cette époque en effet, les procédures d'oralisation du texte écrit passent par la mise en valeur d'une voix singulière.

L'apparition de formes apparentées au DIL dans des corpus de la première modernité a donc de quoi surprendre et pose à la stylistique historique une question de méthode : s'agit-il d'une collection de traits formels réunie par hasard et que l'œil du lecteur contemporain, acculturé à des pratiques ultérieures de la langue littéraire, reconnaît de manière anachronique, ou d'un véritable réseau constituant un patron ?

\section{4- Le repérage du DIL ancien dans la variation des pratiques du discours rapporté}

D'une manière générale, le repérage d'une séquence de DIL dans les corpus d'Ancien Régime est favorisé au sein d'un contexte narratif faisant déjà intervenir le discours d'autrui et caractérisé par une rhétorique de la variation. Bally note ce point dans l'article de 1912 où il formalise les traits du style indirect libre : « Les divers styles, en se succédant, se servent mutuellement de contrôle et toute fausse interprétation est exclue ${ }^{\text {xvi }}$ ». La séquence affichant certains traits du DIL intervient comme une modulation du discours autre substituant au discours indirect ou narrativisé une forme qui associe le système temporel et personnel du locuteur citant, avec des traits modaux et stylistiques assumés par le locuteur cité.

Facilement repérable, la présence d'une modalité d'énonciation exclamative ou interrogative n'est pas un indice particulièrement probant pour repérer un DIL dans les corpus d'Ancien-Régime. Ces modalités marquées sont en réalité souvent assumées par le narrateur dans le cadre d'une figure pathétique. Autre possibilité, le point d'interrogation peut être utilisé à la fin d'une interrogative indirecte avec ellipse du verbe recteur, comme dans cet exemple extrait d'un roman de Jean-Pierre Camus :

[1-Camus, L'Illustre parisienne (1641)]

Il luy fit voir par des lustres formez, par l'artifice d'une subtile eloquence, dont le monde n'est que trop pourveu, et qui seroit mieux nommée causerie ou cajollerie ; que les loix Civiles et les Canoniques, les Humaines et les Divines n'estoient pas si contraires, qu'elles ne se peussent concilier, que Dieu etc. Qu'il y avoit des fausses vertus aussi bien que des happelourdes entre les pierreries; que tout ce qui a du lustre n'est pas de l'or, que le Cristal paroist comme un Diamant, bien qu'il n'en ait ny la valeur, ny la fermeté : Que l'impiété se masque quelquefois du nom et de l'apparence de piété ; que plusieurs confessent Dieu par la bouche qui le nient par les effects. Et quels effects plus funestes que de reduire ses parens avant terme au tombeau ? leur faire traisner une fin de vie triste, solitaire, languissante, et pire que mille morts, que de desoler une famille, perdre un bien, qui bien mesnagé et employé pour le service de Dieu pourroit conduire son possesseur au ciel aussi bien qu'une pauvreté affectée : Que les exercices de la justice valoient bien ces menées et les emplois dont l'on occupe et amuse plusieurs Moines dans les Convens $s^{\mathrm{x} v i i i}$.

La séquence de discours indirect est introduite par le verbe « luy fit voir [...] », suivi du conjonctif que. La reformulation opérée par la voix narrative est signalée par l'emploi des imparfaits (qui, au sein d'un discours direct, auraient été actualisés par un présent gnomique) et par le retour de la modalité assertive qui efface le marquage subjectif de l'énoncé. L'apparition de deux verbes au présent («se masque», 
« confessent ») introduit une première variation analysable comme un fait de polyphonie énonciative : le discours indirect du narrateur est contaminé par la parole qu'il représente. Dans un tel contexte, l'interrogation rhétorique ne s'autonomise pas de la séquence de discours indirect au sein de laquelle elle s'insère : les compléments du comparatif, en effet, s'accumulent au-delà du point d'interrogation et sont réintégrés dans la modalité assertive du discours indirect. Il semble donc qu'on ait plutôt affaire à une interrogative indirecte ponctuée par un point d'interrogation, phénomène très fréquent au XVII siècle. Il reste que le segment se démarque au sein de la séquence : les ellipses du verbe recteur et du verbe subordonné ainsi que l'amplification des compléments à l'infinitif lui confèrent un style plus inactuel, comme si l'énoncé du discours autre glissait dans un espace fictionnel que le narrateur tenait à la fois à représenter et à mettre à distance.

Cette tendance à la désactualisation se retrouve dans des modalités interrogatives relevant cette fois sans ambiguïté du DIL :

[2-La Fayette, La Princesse de Clèves, 1678]

Elle s'enferma seule dans son cabinet. De tous ses maux celui qui se presentoit à elle avec le plus de violence, estoit d'avoir sujet de se plaindre de Monsieur de Nemours, et de ne trouver aucun moyen de le justifier. Elle ne pouvoit douter qu'il n'eust conté cette avanture au Vidame de Chartres ; il l'avait avoüé, et elle ne pouvoit douter aussi, par la manière dont il avait parlé, qu'il ne sceust que l'aventure la regardoit. Comment excuser une si grande imprudence, et qu'estoit devenue l'extrême discretion de ce Prince, dont elle avoit esté si touchée ? Il a esté discret disoit-elle, tant qu'il a crû estre malheureux ; mais une pensée d'un bonheur, mesme incertain, a finiy sa discretion ${ }^{\mathrm{xix}}$.

Introduite par la forme pronominale «se présentoit à elle», la séquence s'interprète jusqu'à «la regardoit » comme un " psychorécit». Dorrit Cohn définit cette forme comme un « discours du narrateur sur la vie intérieure d'un personnage ${ }^{\mathrm{xx}} »$, et elle la distingue du monologue intérieur narrativisé qui relève quant à lui du DIL ${ }^{x x i}$. L'une des marques caractéristiques de cette forme est la présence de verbes d'opération mentale et d'imparfaits à valeur subjective. Dans ce contexte, l'interrogative marque une solution de continuité puisqu'on passe de l'énonciation analytique et distanciée du narrateur à une modalité qui marque l'incertitude du personnage et implique donc un changement d'énonciateur. Ce passage au DIL est également marqué par l'usage de l'infinitif " comment excuser », qui désactualise l'énoncé et accuse l'altérité du discours représenté.

Le repérage du DIL, plus discret en l'absence de modalités d'énonciation marquées, est rendu possible par des syntagmes prépositionnels d'attribution ainsi que des indices stylistiques :

[3-A. Mareschal, Chrysolite, 1627]

Toutesfois ce ne fut point sans luy en faire quelques reproches; les pendans qu'elle avoit receus luy pesoient plus sur le cour qu'à elle mesme aux oreilles; ce n'estoit pas à une fille telle qu'elle estoit, de prendre rien d'un homme à qui elle ne vouloit rien donner ; c'estoit à son avis trop de licence et à l'un et à l'autre ; qu'il y avoit des marques en amour, qu'on avoit autant moins de grace à recevoir, qu'il y en avoit à les presenter ${ }^{x x i i}$.

La séquence s'ouvre sur un verbe de discours narrativisé régissant un syntagme pronominal « luy en faire quelques reproches ", suivi d'un point-virgule. S'ensuit un enchaînement de phrases à l'imparfait et au plus que parfait ponctuées à chaque fois par un point-virgule. Ces propositions pourraient être interprétées comme des éléments strictement narratifs indiquant les causes des «reproches» du personnage, conformément à la valeur d'arrière-plan de l'imparfait, si le syntagme prépositionnel «à son avis » ne venait pas désambiguïser le statut énonciatif de l'énoncé. L'apparition d'une subordonnée introduite par que, qu'on peut analyser ici comme une complétive ayant pour antécédent les " reproches », assure la même fonction de rattachement de l'énoncé à une source énonciative seconde, distincte du narrateur. La rhétorique brève et expressive qui caractérise la séquence permet également d'identifier l'énonciateur comme étant le personnage et non l'instance narratrice. L'effet de brièveté est assuré par l'enchaînement de prédications peu développées et juxtaposées au moyen de points-virgules, procédé caractéristique du style dit coupé. L'expressivité est marquée par les figures de parallélisme ( 1 le cœur » vs «les oreilles », "prendre » vs « donner »). Ce style formulaire caractérise la rhétorique passionnée de son énonciateur et fonctionne sans doute, pour un lecteur d'Ancien Régime acculturé aux différents registres (genera dicendi) codifiés par la rhétorique, comme l'indice remarquable d'une parole orale et plaintive. 
La présence d'incises à l'imparfait assume cette même fonction de désambiguïser des formes de discours rapporté flottantes :

[4-J.-J. Rousseau, Julie ou la nouvelle Héloïse, 1761]

Claire pretendit remarquer que tu ne faisois pas une meilleure figure; tu lui paroissois embarrassé de ta contenance, inquiet de ce que tu devois faire, n'osant aller ni venir, ni m'aborder ni t'éloigner, et promenant tes regards à la ronde pour avoir, disoit-elle, occasion de les tourner sur nous. Un peu remise de mon agitation, je crus m'apercevoir moimême de la tienne $[\ldots]^{\text {xxiii }}$

Sans l'incise, rien ne nous permettrait de savoir que la séquence allant de « tu lui paroissois » jusqu'à « de les tourner sur nous » relève du discours rapporté et non du simple marquage d'un point de vue au sein d'un énoncé narratif: les différentes actions de Saint-Preux seraient dans ce cas décrites et appréhendées du point de vue de Claire, ce qui expliquerait la présence de l'imparfait. On observe toutefois des traits stylistiques déjà présents dans les occurrences précédentes : la brièveté du style coupé et la désactualisation opérée par les formes non personnelles du verbe («n'osant aller ni venir, ni m'aborder ni t'éloigner ») sont des indices de polyphonie que l'incise ne fait que confirmer.

L'observation des occurrences nous conduit à poser l'hypothèse qu'il existe bien, aux XVII ${ }^{\mathrm{e}}$ et $\mathrm{XVIII}^{\mathrm{e}}$ siècles, un patron stylistique du DIL, même si les occurrences en sont rares. Elles interviennent dans des séquences narratives au sein desquelles la représentation du discours d'autrui tient le devant de la scène, et elles présentent l'intérêt de s'intégrer aisément au flux de la narration tout en arborant des marques d'expressivité. Des séquences formellement identiques à des énoncés narratifs ou descriptifs seront ainsi reconnues comme des discours rapportés en raison de leur agencement stylistique remarquable :

[5-F. de Rosset, Histoires tragiques, 1619]

Chacun d'eux prend une chaise ou il s'assied, et la Damoiselle s'assied en une autre aupres d'eux. Ils ne cessent de la contempler, et de l'admirer. L'un loue son front, et dit que c'est une table d'yvoire bien polie. L'autre s'arreste sur ses yeux, et assurent que ce sont les deux flambeaux dont Amour allume toutes les ames genereuses. L'autre se met sur la louange de ses blonds cheveux qu'elle delioit, parce qu'il estoit temps de s'aller coucher, et ne cesse de proferer tout haut, que ce sont les filets où le fils de Cypris arreste la liberté des hommes et des Dieux. Enfin il n'y a partie en son corps qu'ils ne prisent. Ses mains ne vont jamais en vain à la conqueste. Sa gorge surpasse la blancheur de la neige : et les petits Amours voletent à l'entour de ses joues, pour y succer les roses, les lys, et les oillets que la Nature y a semez. Apres qu'ils ont bien chanté ses perfections, elle se leve de sa chaire, s'approche du feu, et puis se tournant vers eux leur tient ce discours: Vous croyez (dict-elle) avoir fair faict un grand gain, d'avoir obtenu de moy l'accomplissement de vos desirs. Il n'est pas si grand que vous penseriez bien. Avec qui pensez-vous avoir eu affaire xxiv?

Cette narration est différente des exemples précédents dans la mesure où elle est actualisée par un présent de narration qui écrase toute structuration des plans du récit, à la différence des occurrences précédentes où l'on observait une alternance imparfait-passé simple. Le discours narrativisé («l'un loue son front», «l'autre s'arreste sur ses yeux », «l'autre se met sur la louange ») est suivi d'une séquence où les verbes métadiscursifs sont absents, et l'on pourrait penser qu'il s'agit d'un portrait élogieux assumé par la voix narrative. Cette interprétation semble toutefois infirmée par la clôture de la séquence et le retour d'une indication métadiscursive ( «apres qu'ils ont bien chanté ses perfections »), qui recatégorise l'ensemble de l'énoncé au présent comme relevant du discours rapporté. Mais surtout, le style coupé de la séquence et sa forte ornementation (métaphores de la «blancheur», des "Amours» et des fleurs) souligne l'altérité énonciative de ces prédications. Le lecteur ne reçoit plus un discours simplement résumé par la voix narrative, comme c'était le cas avec le discours narrativisé : on ne lui raconte plus seulement que telle ou telle parole a été prononcée ou pensée, on lui montre comment elles ont été énoncées.

La configuration formelle du DIL d'Ancien Régime pose bien sûr la question de son interprétation pragmatique : en particulier, on se demandera à quelle visée répond sa tendance à la brièveté et à l'expressivité. 


\section{5-Pragmatique du discours indirect libre de la première modernité}

L'utilisation du DIL, et en particulier de sa forme endophasique, dans les textes fictionnels de la fin du $\mathrm{XIX}^{\mathrm{e}}$ siècle est généralement interprétée comme symptomatique du tournant subjectiviste de cette époque littéraire. L'énoncé narratif se déroule tout seul, sans la présence médiatrice d'un narrateur, et devient l'appareil enregistreur des paroles et du flux de conscience des personnages ${ }^{\mathrm{xxv}}$. Dans nos occurrences, en revanche, la voix narrative n'est pas totalement effacée : on perçoit le travail de reformulation et d'abréviation que cette instance opère sur le discours qu'elle représente. Le DIL d'Ancien Régime s'analyse donc comme une pratique bivocale du discours rapporté au sein de laquelle l'instance narratrice est plus ou moins présente, même si, dans certains cas, celle-ci peut être particulièrement discrète.

Le style coupé du DIL et sa tendance générale à la brièveté s'explique par l'intention résomptive qui caractérise la forme : la voix narrative entend donner du discours autre une représentation abrégée, selon un mode qui se distingue pourtant du discours indirect et du discours narrativisé. Dans ces deux autres formes, en effet, l'information apportée concerne respectivement l'événement du discours autre (le fait qu'untel a dit) et le contenu propositionnel du discours autre (ce que untel a dit). Le DIL, lui, aura plutôt tendance à mettre en évidence le style du discours d'autrui, en sélectionnant les traits stylistiques les plus marquants de ce dernier. En donnant du discours d'autrui une représentation stylisée et abrégée, il rend compte de la manière propre du locuteur cité et des traits caractéristiques qui permettent de garder ses paroles en mémoire. C'est la raison pour laquelle les séquences de DIL font apparaître des propositions relativement courtes marquées par un trait de style dominant, comme par exemple la métaphore pétrarquiste dans l'exemple 5 ( «la blancheur de la neige », «les petits Amours»). Ces traits sont présentés par l'instance narratrice comme des images représentatives de la manière de parler du personnage. Le DIL relève à ce titre d'une procédure de caractérisation : en effet, dans la perspective rhétorique de l'Ancien Régime, le style va de pair avec l'expression d'un caractère : tel style métaphorique sera l'expression d'un caractère amoureux, colérique, menteur, puéril. Le DIL peut, de ce fait, prendre une valeur d'exemplification, assimilable au portrait de mœurs tel qu'il se pratique dans le genre satirique :

[6-P. Scarron, Le roman comique, 1641]

La Rancune donc estoit de ces Misantropes qui haissent tout le monde, et qui ne s'aiment pas eux mesmes, et j'ay sçeu de beaucoup de personnes, qu'on ne l'avoit jamais veu rire. Il avoit assez d'esprit, et faisoit assez bien de meschans Vers ; d'ailleurs nullement homme d'honneur, malicieux comme un vieil Singe, et envieux comme un chien. Il trouvoit à redire en tous ceux de sa profession. Belleroze estoit trop affecté. Mondory rude, Flodory trop froid, et ainsi des autres ; et je croy, qu'il eust aisément laissé conclure, qu'il avoit esté le seul Comédien sans défaut ; et cependant, il n'estoit plus souffert dans la troupe, qu'à cause qu'il avoit vieilli dans le mestierxxi.

Le narrateur place sous les yeux de son lecteur un portrait observé à la loupe des médisances les plus marquantes de son personnage, en représentant les formules les plus remarquables de ce dernier. L'usage de l'imparfait s'explique donc par le caractère subjectif et mémoriel des procès qui sont décrits, ce qui rapproche le DIL d'Ancien Régime de son usage moderniste comme « discours perçu » tel que l'a analysé Gilles Philippe ${ }^{\mathrm{xxvii}}$. Les paroles représentées ne sont pas envisagées comme des contenus propositionnels mais comme des manières de dire qui ont marqué la mémoire du locuteur.

Dans certaines occurrences, le caractère subjectif et mémoriel du DIL est thématisé dans le contexte immédiat de son apparition par de nombreux indices de focalisation interne :

[7-O. S. de Claireville, Le Gascon extravagant, 1639]

Lors que je commençois à m'ennuyer d'entendre tout le monde discourir, je venois à la traverse, $\&$ disois ma ratelée comme les autres. Chacun y avoit son inclination \& son humeur apart, $\&$ apres avoir discouru des afaires \& des personnes, on se jetoit dessus une autre matiere. L'autorité des Histoires y estoit confrontée, chacun donnoit son avis, les defauts de la Republique estoient reparez par la fantaisie de celuy qui n'aprouvoit pas le concours : \& finalement j'y entendois une harmonie semblable au ramage de plusieurs Oyseaux. On y discouroit de la paix, [p. 346] de la guerre, du trafic, des voyages, \& de tout ce qu'on se peut imaginer. Quelqu'un qui vouloit passer pour serieux, pesoit ses mots en parlant, usoient [sic] de termes graves, alleguoit les passages, louoit un livre, improuvoit l'autre, \& quelque delicat que fust un Auteur, il y trouvoit pourtant tousjours quelque chose qui luy 
escorchoit l'oreille, une periode le choquoit, une pensée n'y estoit pas couchée assez à propos : il y avoit trop de vanité : ce n'estoit qu'un discours naturel : il n'y avoit rien à aprendre : c'estoit dommage d'avoir gasté du papier pour si peu de chose : il s'estonnoit commentil se trouvoit des personnes qui fissent estat de cét Auteur ${ }^{x x v i i i}$.

Dans cette narration à la première personne, l'ensemble des discours représentés sont englobés dans une focalisation interne qui appréhende les discours d'autrui du point de vue du narrateur. Ce processus est amorcé par la représentation à l'imparfait du discours narrativisé et sa passivation : « l'autorité des Histoires y estoit confontée », « les defauts de la Republique estoient reparez ». La représentation de la perception par le narrateur des discours d'autrui se substitue à celle de leur énonciation, phénomène qui aboutit à l'effacement textuel des locuteurs cités, à travers la forme passive puis les expressions référentielles indéfinies «on» et «quelqu'un». On entre ainsi progressivement dans la subjectivité du narrateur qui perçoit les discours prononcés autour de lui que comme le «ramage de «plusieurs Oyseaux ». Cette perception apparaît donc comme une représentation confuse, approximative et hyperstylisée des propos qui ont été tenus.

Dans les deux exemples précédents, l'intention résomptive du DIL s'accompagne, de la part du narrateur, d'une visée satirique, la mémoire de ce dernier ayant sélectionné les traits représentatifs ridicules du discours perçu. D'une manière inverse, mais qui repose sur le même ancrage mémoriel du locuteur citant, le DIL peut obéir à une visée empathique. Dans l'exemple n² (La Fayette), la modulation du psychorécit par une interrogation marque l'abolition d'une distance entre les passions du nrrateur et celles du personnage. Autrement dit, la mise en évidence des traits caractéristiques du discours autre opérée par le DIL peut fonctionner aussi bien sur le principe d'une distance que d'une fusion entre les voix.

\section{Conclusion}

Le DIL apparait bien comme une forme de discours rapporté attestée dans les textes de la première modernité au moyen d'un patron stylistique aux traits reconnaissables. Son absence dans les traités de grammaire classiques s'explique par la rareté de ses occurrences, et par le fait qu'il ne possède pas de configuration syntagmatique stabilisée en langue. Défini par la coprésence ponctuelle d'une énonciation narratoriale et des traits modaux et stylistiques appartenant à un discours autre, il se rend remarquable au sein de l'énoncé par une tendance à la brièveté et à la désactualisation. Peut-être qu'en cherchant du côté des définitions rhétoriques de la description, de l'ekphrasis ou encore de l'hypotypose, on pourrait trouver des traces, sinon d'une théorisation de cette forme, du moins de certains procédés de caractérisation qui s'en rapprocheraient. Il n'est pas impossible non plus que cette manière de représenter les discours et les pensées ait été thématisée au chapitre des lieux communs (au sens technique du terme), en particulier ceux des moun ou des passions.

La spécificité de la forme du DIL au sein du système des discours rapportés tient donc à sa bivocalité, mais l'usage qui en est fait sous l'Ancien-Régime est différent de celui de la fin du XIXe siècle car la conscience du narrateur y tient lieu de mémoire affective, qui se souvient de propos marquants et s'efforce d'en donner un portrait ressemblant.

Le DIL apparaît ainsi au service d'un besoin spécifique du métalangage, celui de présenter une image du style des autres, et non pas seulement de leur propos. Voir qu'une telle forme est attestée dans le roman d'Ancien Régime semble confirmer l'approche Bakhtinienne d'une dimension foncièrement réflexive du discours romanesque et de la vocation du genre à donner du monde une représentation polyphonique, mais cette pratique littéraire se nourrit d'une aptitude fondamentale du langage à s'observer lui-même par le biais du discours des autres. Aussi serait-il utile de se pencher sur les pratiques du discours indirect libre qui existent dans les autres genres de discours de l'époque classique.

\section{Bibliographie}

Arnauld, A., Lancelot, Cl., Grammaire générale et raisonnée : contenant les fondements de l'art de parler, Paris, P. Le Petit, 1660.

Authier-Revuz, J., Ces mots qui ne vont pas de soi. Boucles réflexives et non-coïncidences du dire, 2 tomes, Paris, Larousse, 1995.

Authier-Revuz, J., « Le discours rapporté », dans R. Thomassone (dir.) Une langue : le français, Paris, Hachette, 2001, p. 192-201.

Authier-Revuz, J., La représentation du discours autre : principes pour une description, Berlin, De Gruyter, à paraître. 
Bally, Ch., «Le style indirect libre en français moderne II », Germanisch-Romanische Monatsschrift, IV/11, 1912, p. 597-606.

Banfield, A., Phrases sans parole. Théorie du récit et du style indirect libre, trad. Cyril Veken, Paris, Seuil, 1985.

Berthonneau, A.-M., Kleiber, G., «Pour une nouvelle approche de l'imparfait : l'imparfait anaphorique méronomique », Langages, 1993/12, p. 55-73.

Camus, J.-P., La Pieuse Jullie. Histoire parisienne [1625], Rouen, Jean De La Mare, 1641.

Caussin, N., Eloquentiae sacrae et humanae parallela libri XVI, Auctore P. Nicolao Caussino, Paris, S. Chappelet, 1619.

Claireville, O. S. de, Le Gascon extravagant, Paris, Cardin Besogne, 1639.

Cohn, D., La transparence intérieure : modes de représentation de la vie psychique dans le roman [1978], trad. A. Bony, Paris, Éditions de Seuil, 1981.

Fabri, P., Le Grand et vray art de pleine Rhetorique utille, profitable et nécessaire à toutes gens qui desirent à bien élégantement parler et escrire, Paris, D. Janot, 1534.

Jaubert, A., La lecture pragmatique, Paris, Hachette, 1990.

La Fayette, Madame de, La Princesse de Clèves, Paris, C. Barbin, 1678.

Lips, M., Le style indirect libre, Paris, Payot, 1926.

Maingueneau, D., Philippe, G., «Les conditions d'exercice du discours littéraire », dans E. Roulet et M. Burger (dir.), Les modèles du discours au défi d'un dialogue romanesque, Nancy, Presses Universitaires de Nancy, 2002, p. 272-283.

Mareschal, A., La Chrysolite ou le secret des romans par le Sieur Mareschal, Paris, T. du Bray, 1627.

Philippe, G., « Le discours indirect libre et la représentation du discours perçu », dans Fabula / Les colloques, Marges et contraintes du discours indirect libre, 2016.

Philippe, G., «Quelques réflexions sur l'expression de stylistique historique », dans Cl. Badiou-Monferran (dir.), La littérarité des Belles-Lettres ; un défi pour les sciences du texte? Paris, Garnier, 2013, p. 272283.

Philippe, G., Zufferey, J., "Cent ans après ", [article paru en ligne], Fabula / Les colloques, Marges et contraintes du discours indirect libre, 2016.

Pomey, Fr., Candidatus rhetoricae, seu aphtonii progymnasmata [1661], Münich, J. W. Schell et J. Wagner, 1664.

Reggiani, Chr., "L'intériorisation du roman : brève histoire du style indirect libre », dans Gilles Philippe et Julien Piat (dir.), La langue littéraire. Une histoire de la prose en France de Gustave Flaubert à Claude Simon, Paris, Fayard, 2009, p. 122-134.

Rodriguez Somolinos, A., "Énonciation et discours rapportés dans les Fables de La Fontaine », Bulletin hispanique, CVII, 2005/1, p. 139-154.

Rosier, L., Le Discours rapporté en Français, Paris, Éditions Ophrys, 2008.

Rosset, Fr. de, Les Histoires memorables et tragiques de ce temps, Paris, P. Chevalier, 1619.

Rousseau, J.-J., Julie ou la nouvelle Héloïse [1761], Jean-Jacques Rousseau. Euvres complètes, t. II, édit. B. Gagnebin, M. Raymond, H. Coulet, Ch. Guyot, J. Scherer, Paris, Gallimard, coll. « Bibliothèque de la Pléiade », 1961.

Scarron, P., Le Romant comique [1651], Lyon, Jean Bruyset, 1678.

Siouffi, G., Le génie de la langue française. Essai sur les structures imaginaires de la description linguistique à l'âge classique, Paris, Honoré Champion, 2010.

\footnotetext{
i [G.Philippe, J. Zufferey « Cent ans après », Marges et contraintes du discours indirect libre, (2016)]

ii [C. Reggiani, «L'intériorisation du roman : brève histoire du style indirect libre », in G. Philippe et J. Piat (dir.), La langue littéraire. Une histoire de la prose en France de Gustave Flaubert à Claude Simon, (2009 : 122-134)].

iii [J. Authier-Revuz, « Le discours rapporté », in R. Thomassone (dir.) Une langue : le français, Paris, Hachette, (2001, p. 192-201:201)]

iv [Ch. Bally, « Le style indirect libre en français moderne II », Germanisch-Romanische Monatsschrift, IV/11 (1912), p. 597-606.1912] [A. Banfield, Phrases sans parole. Théorie du récit et du style indirect libre, (1985)]
} 
iv [Voir notamment Ch. Bally (1912) et [M. Lips, Le style indirect libre (1926)]

v [Voir notamment Ch. Bally (1912) et [M. Lips, Le style indirect libre (1926)]

vi [Voir J. Authier-Revuz, La représentation du discours autre : principes pour une description, à paraître. Je remercie chaleureusement l'autrice de cet ouvrage pour m'avoir permis de le lire avant sa publication, et pour les indications précieuses qu'elle m'a données au cours de la rédaction de la présente étude.]

vii [G. Philippe, «Quelques réflexions sur l'expression de stylistique historique », in C. Badiou-Monferran (dir.), $L a$ littérarité des Belles-Lettres ; un défi pour les sciences du texte? (2013 : 272-273)]

viii [D. Maingueneau et G. Philippe, «Les conditions d'exercice du discours littéraire », dans E. Roulet et M. Burger (dir.), Les modèles du discours au défi d'un dialogue romanesque (2002:366-367)]

ix [L. Rosier, Le Discours rapporté en Français (2008:21)]

x [A. Arnauld, C. Lancelot Grammaire générale et raisonnée (1660:112-113)]

xi [L. Rosier, Le Discours rapporté en Français (2008 : 12)]

xii [P. Fabri Le Grand et vray art de pleine Rhetorique utille, profitable et nécessaire à toutes gens qui desirent à bien élégantement parler et escrire (1534:185)]

xiii [N. Caussin, Eloquentiae sacrae et humanae parallela $(1619: 327)]$ Nous traduisons.

xiv [F. Pomey, Candidatus rhetoricae (1664:259)]

xv [F. Pomey, Candidatus rhetoricae (1664:261)] Nous traduisons.

${ }^{x v i}$ [G. Siouffi, Le génie de la langue française. Essai sur les structures imaginaires de la description linguistique à l'âge classique (2010: 165)]

xvii Cette variation des modes de discours rapporté est notamment représentée chez La Fontaine: voir [A. Rodriguez Somolinos, «Énonciation et discours rapportés dans les Fables de La Fontaine », Bulletin hispanique, CVII, 1, (2005: 139-154)]

(2005)]

xviii [J.-P. Camus, La Pieuse Jullie. Histoire parisienne (1641: 84-87)] Ici et dans les citations suivantes, nous soulignons.

xix [La Fayette, La Princesse de Clèves, (1678: III, 85)]

xx [D. Cohn, La transparence intérieure (1981:28)]

xxi D. Cohn définit le monologue intérieur narrativisé comme le « discours mental d'un personnage pris en charge par le discours du narrateur $»:(1981: 29)$.

xxii [A. Mareschal, La Chrysolite (1627: 182)]

xxiii [J.-J Rousseau, La Nouvelle Hélö̈se (1761 : I, 23)]

xxiv [F. de Rosset, Histoires tragiques (1619 : X, 340-341)]

${ }^{x x v}$ Voir Ch. Bally (1912), Banfield (1985), Reggiani (2009), Philippe et Zufferey (2016).

xxvi [P. Scarron, Le Romant comique (1641: I, v, 20-21)]

xxvii [G. Philippe, « Le discours indirect libre et la représentation du discours perçu » (2016)]

xxviii [O. S. de Claireville, Le Gascon extravagant, (1639 : 346-347)] 